\title{
Deconstructing the performance of everyday activities: a case in dementia
}

\author{
Clarissa M. Giebel ${ }^{1,2, *}$ and Daniela Montaldi ${ }^{2}$ \\ ${ }^{1}$ School of Health Sciences, University of East Anglia, Norwich, UK \\ 2 Division of Neuroscience and Experimental Psychology, The University of Manchester, UK
}

*Correspondence should be addressed to: Clarissa Giebel, Queen's Building, School of Health Sciences, University of East Anglia, NR4 7TJ, Norwich, UK. Email: C.giebel@uea.ac.uk; Telephone: +441603593259 


\section{ABSTRACT}

Background: The assessment of everyday functioning in dementia currently is very global and in most cases fails to provide an in-depth overview of specific areas of deficits. Every activity comprises different sub-tasks which may be impaired to different degrees. Performance of some sub-tasks might be maintained and could thus be the foundation for remaining independent for longer. Thus, the objective of this study was to explore the benefits of breaking down everyday activities into sub-tasks.

Methods: A total of 183 family carers of people with mild dementia completed the revised Interview for Deteriorations in Daily Living Activities in Dementia 2 (R-IDDD2) rating their relative's everyday functioning. Each of the 20 activities comprised three sub-tasks. Data were analysed using ANOVA with Bonferroni corrections, and sub-tasks were clustered in relation to different forms of cognition.

Results: The majority of activities showed at least one major area of impairment. Sub-tasks could be clustered based on different types of cognition. Several sub-tasks had a focus on memory (forgetting it is time to do the cleaning; forgetting previously known telephone numbers), whereby short-term, long-term, and prospective memory could be distinguished further. Other sub-tasks were clustered into attention (getting more distracted when driving) and executive function (sorting out bills).

Conclusions: The R-IDDD2 and its analysis of sub-task performance offers a novel platform to examine impairments comprehensively. This can help both in aiding timelier diagnosis by recognising subtle deficits, but also in care management planning, whereby family and paid carers should only care for those sub-tasks that are most impaired and thus encourage remaining independent for longer.

Key words: dementia; activities of daily living 


\section{INTRODUCTION}

Difficulties in initiating and performing complex instrumental activities of daily living (IADLs) and basic ADLs is a major symptom of dementia (Giebel et al., 2016a; Monaci and Morris, 2102). To date, a great deal of research has focused on more global assessments of this symptom (i.e. O'Connor et al., 2016; Pocnet et al., 2013). However, with a push to improve the recognition of symptoms and timelier diagnosis rates of dementia, understanding better how individual activities deteriorate throughout dementia is vital. Therefore, understanding how individual sub-tasks of each IADL and ADL deteriorate can provide an even better framework to recognise subtle changes.

To date, little research has broken down everyday activities into their sub-tasks. A sub-task represents one element of an individual activity, meaning that one activity constitutes several subtasks. For example, 'sorting out bills' is one sub-task of the activity of finance management. Findings on the DAD scale (Gelinas et al., 1999) allows the assessment of individual sub-tasks of certain few activities (Mioshi et al., 2007), whilst research generally fails to evaluate these sub-tasks individually. Giovannetti and colleagues $(2006 ; 2015)$ for example have explored in depth the different steps and action errors that people with dementia make when making a toast or preparing a hot drink; wrapping a gift; and packing a lunch box or picnic basket - three activities part of the Naturalistic Action Test (Schwartz et al., 2003), whilst finance management is also one of the most explored IADLs in dementia. The Financial Capacity Instrument suggests that finance management comprises nine subactivities, of which basic monetary skills and asset knowledge are found to be best maintained in mild dementia (Chiong et al., 2014; Griffith et al., 2003; Marson et al., 2000). Not every activity can be broken down into nine sub-activities however. Indeed, with a large number of IADLs, ranging from eight (Lawton and Brody, 1969) to 47 (Patterson et al., 1992), assessing nine sub-tasks for every IADL would not be feasible. Thus, we designed a scale containing 20 everyday activities, each of which contain three sub-tasks. This scale is called the revised Interview for Deteriorations in Daily Living Activities in Dementia 2 (R-IDDD2) (Giebel et al., 2016b).

Breaking down everyday activities can also help in further establishing the relationship with cognition (Schmitter-Edgecombe and Parsey, 2014; Shankle et al., 2013; Vermeersch et al., 2015). Although research into different types of cognition and their impact on everyday functioning is in its infancy, not only memory, but also executive function and attention for example have been shown to be associated with successful performance of everyday tasks (Martyr and Clare, 2012; Yamin et al., 2016). Memory however is such a vast term that comprises a number of different sub-types, including long-term, short-term, and prospective memory. Each type processes information differently, which is why the link between 'memory' and everyday functioning needs to be explored more comprehensively. This can be achieved by breaking down tasks into sub-tasks, which can then provide some guidance as to whether an impairment lies with remembering to do something in the future (prospective memory), remembering something from the past (long-term memory), or being easily distracted (attention).

Such an activity breakdown can have important implications for everyday practice. Whilst a person with dementia $(P w D)$ might experience some difficulties with an activity, such as using the telephone, there might be some sub-tasks of the activity that are less affected. In care management 
planning, but also for immediate family caring for the PwD, it is important that the informal or formal carer does not care for the entire activity, but leaves the PwD to perform those sub-tasks that he or she is still able to do. Carers should only directly address those specific sub-tasks that are impaired the most, and training should be provided for these. Hence, breaking down IADLs into their individual components can help in guiding the care plan whilst still ensuring that independence is maintained by the PwD themselves, where possible.

The objective of this study was to explore whether people in mild dementia display subtle and more pronounced deficits within individual everyday activities. Improved knowledge of the intrinsic deficits of individual everyday activities in mild dementia can help to recognise and diagnose dementia timelier. This knowledge can have further implications for effective care management planning and intervention design. Without previous data on sub-task performance, no hypotheses were made.

\section{METHODS}

\section{Participants}

Informal carers were eligible to take part in the study if they cared for someone in the mild stage of dementia. This was defined as having a Mini-Mental State Examination (Folstein et al., 1975) score of 21 or above (Earnst et al., 2001), a Montreal Cognitive Assessment (Nasreddine et al., 2005) score of 15 or above (van Steenoven et al., 2015), or an equivalent score on the Addenbrooke's Cognitive Examination III (Hsieh et al., 2013).

\section{Materials}

The R-IDDD2 (Giebel et al., 2016b) assesses the initiative and performance of everyday activities and is based on the original IDDD (Teunisse et al., 1991) and the first stage of the revised version, the RIDDD (Giebel et al., 2016a). The scale comprises 17 activities on the initiative scale and 20 on the performance scale. Each activity can be rated on a Likert scale from ' 0 ' ('never lacking motivation/ never any difficulties') to '4' ('always lacking motivation/ always difficulties'). On the performance scale, each activity comprises three sub-tasks. Participants are asked to rate the performance on all three sub-tasks if the overall activity is rated as impaired between ' 1 ' and ' 4 '.

The R-IDDD2 is shown to have high internal consistency for its initiative subscale for basic ADLs (including washing oneself, dressing, and brushing hair/teeth) ( $\alpha=0.894)$ and IADLs $(\alpha=0.934)$, and for its performance subscale for basic ADLs $(\alpha=0.858)$ and IADLs ( $\alpha=0.959)$ (Giebel et al., 2016b).

Table 1 provides an overview of the 20 activities and their respective sub-tasks.

\section{[insert here Table 1]}




\section{Procedure}

Informal carers of people with mild dementia were approached by staff at nine NHS Trusts across England, as well as being recruited via the JoinDementiaResearch Network. Carers received the questionnaire during a routine visit with their relative with dementia, or via post, and could complete the questionnaire in their own time. The questionnaire lasted approximately 20 to 30 minutes to complete, and all carers received a free-return envelope to post the completed questionnaire back to the research team. Ethical approval was obtained prior to study begin from the NRES Committee North West - Preston (14/NW/0241). Data were collected between April 2014 and October 2015.

\section{Data analysis}

Data were analysed using SPSS 22 for Windows. Frequency analysis was used to calculate the proportion of PwD impaired in each activity and the proportion with severe impairments (scoring 'often' or 'always') on each sub-task. Paired samples t-tests were performed to explore variations in sub-tasks for each of the 20 performance activities.

Each of the 60 sub-tasks was clustered together based on their implied underlying cognitive underpinnings, including attention, executive function, as well as prospective, recent, and remote memory. The operational definitions of these types of cognition were anchored in neuropsychology and defined in the context of everyday behaviour: Attention was considered in its wider sense, by not specifying a particular type such as divided or selective attention, but by referring to the level of focus and constant awareness required in performing a task. Executive function was similarly considered in a broader sense by relating to everyday problems and problem solving skills. Prospective memory referred to remembering to perform a task in the future. Recent memory referred to the memory of a task only just having been performed, or possibly whilst the task is still being performed. Remote memory refers to events in the past, or to activities that have been performed in the past and now performing these cannot be remembered any longer.

Frequency analysis was used to explore the proportion of total sub-tasks impaired, as well as the proportion of impaired sub-tasks in the five clusters of cognition.

\section{RESULTS}

In total, 183 carers completed and returned the R-IDDD2. The majority of carers were female $(75 \%)$, on average $66(+/-11)$ years old, and spouses $(69 \%)$ or adult children $(26 \%)$. Most carers were the only carer for their relative with dementia $(82 \%)$ and lived with their relative $(74 \%)$. People with dementia were mostly male (57\%), on average $77(+/-9)$ years old, and had Alzheimer's disease (62\%). The remaining PwD had diagnoses of vascular disease (12\%), mixed dementia (17\%), or other dementia subtypes including Lewy bodies and fronto-temporal dementia (10\%).

Table 1 shows the proportion of PwD who were impaired on the overall performance of the 20 daily activities. Over $80 \%$ of PwD were impaired handling finances, maintaining an active social life/engaging in hobbies, following current affairs, monitoring own day, and monitoring a current activity. Table 1 also shows the proportion of $\mathrm{PwD}$ who were reported to often or always experience 
difficulties in a particular sub-task. Paired samples t-tests were performed to analyse the sub-task ratings for performance on each activity. Table 2 only shows the results of those t-tests which found significant variations. In total, 16 activities had at least one major area of impairment that varied significantly from the performance of other sub-tasks. Four activities (using the computer, preparing a hot meal, medication management, taking public transport), showed no significant variations across sub-tasks.

Clustering the 60 sub-tasks into their most prominent suggested links with cognition resulted in 17 sub-tasks for remote memory, 15 for executive function, 6 for prospective memory and attention, respectively, and 5 for attention (see Table 3). Eleven sub-tasks could not be categorised.

Of the 16 activities with significant variations across their sub-tasks, the majority of the most impaired sub-tasks had a theme of forgetting and memory in common, either for recent or remote events or for events in the future. Leaving clothes on for too long (Dressing), forgetting to brush hair or teeth or to shave, forgetting where items are located in the shop (Shopping), forgetting previously known telephone numbers, forgetting that it is time to do the cleaning, getting lost (Driving), forgetting what happened at the last meet up of a social activity, and forgetting what the PwD was currently doing (Monitoring an activity) all suggest memory deficits.

For some activities, impaired sub-tasks had a theme of attention, such as getting more distracted (Driving) and being easily distracted (Monitoring an activity).

Some sub-activity impairments could also be clustered together under the theme of executive functioning, with some of the most impaired sub-tasks being sorting out bills (Finance management), only preparing simple meals (Preparing a cold meal), and requiring help to plan the day (Monitoring the day).

The more spared sub-tasks, including correctly setting the table (Cleaning), forgetting how to do key tasks (Hobbies), difficulties recognising family members (Recognising familiar faces), difficulty counting change (Finance management), and awareness of time (Monitoring own day), covered several cognitive domains.

Comparing the proportion of severely impaired sub-tasks between the five cognitive clusters, it emerged that the greatest proportion of impairment was found for executive functioning (36 +/- 21 per cent). If different memories were taken together, memory would be the cognitive function suggested to underlie the largest number of impairments in sub-tasks. Distinguishing between different memory types, remote memory made up the largest proportion of impairments $(19+/-15$ per cent), followed by recent (11 +/- 9 per cent) and prospective memory $(9+/-12$ per cent). A similar proportion to recent and prospective memory was impaired on attention $(9+/-8$ per cent). The majority of PwD showed a spread of impairment across these clusters, with 29 cases showing a clear cluster of impairment with either deficits in only one or two cognitive clusters.

[insert here Table 2 and 3] 


\section{DISCUSSION}

Deficits in everyday functioning in mild dementia cannot only be detected through the assessment of individual activities, but the analysis of sub-tasks, by breaking down each activity into its components, can potentially add much needed detail regarding the nature of the deficit. This exploratory study has clearly shown how people with mild dementia struggle more with performing certain sub-tasks of specific activities, such as 'forgetting where items are in the shop' when going shopping. This information can be critical for non-pharmacological interventions that should be designed to target those specific sub-tasks showing impairment, rather than simply the task as a whole.

In light of significant variations in sub-task performance for the majority of activities, a breakdown of activities can provide important additional detail. Whilst the R-IDDD2 is not the only scale breaking down activities into their initiative and performance, as work by Bier and colleagues (2013) shows activities can be broken down further, into formulating a goal, planning, execution, and verifying goal attainment, the R-IDDD2 addresses novel activities such as computer use and driving, and allows the assessment of their individual sub-tasks. Instead of providing general training to maintain or regain the ability to perform a specific activity, such as maintaining an active social life, targeted therapy can be offered by addressing the most impaired sub-task, in this case 'forgetting what happened at the last social event'. Only four activities showed no differences between sub-task performances, so that in those cases a breakdown might not be as informative. The R-IDDD2 contains three sub-tasks for each activity. Whilst some activities may contain more than three subtasks, such as finance management as evidenced in the Financial Capacity Instrument (Marson et al., 2000), the R-IDDD2 already asks participants to rate the performance of 60 activities, in addition to 17 initiative ratings. Hence, based on qualitative data obtained from carers regarding individual deficits for each activity (Giebel et al., 2016a), three sub-tasks were considered sufficient to not overburden participants but to equally obtain an appropriate breadth of information.

Understanding precisely where the deficits lie in performing a certain task can also help in effective care management planning. Based on the present findings, few PwD put on clothes in the wrong order or required help to put on clothes, whereas more people needed assistance so as to not wear the same clothes for too long. Obviously this needs to be assessed on an individual basis so that care and treatment are personalised. However, the evidence does suggest that carers should only target the issue surrounding leaving the same clothes on for too long, as PwD were significantly more able to perform other sub-tasks. This would delay the PwD becoming increasingly more reliant on family and paid carers, and thus encourage the PwD to remain independent and use those skills that are little compromised. One possible limitation of these findings is that reports were provided by carer proxies, which can vary from directly observed performance (Zanetti et al., 1999). However, the majority of studies into everyday functioning in dementia uses proxy assessments (Mioshi et al., 2007; Nagaratnam et al., 2013; Sikkes et al., 2011), and can indeed be a better estimation of the everyday functioning abilities of the PwD, due to likely anosognosia in the patient.

Amongst those impaired sub-tasks, certain patterns emerged that could be linked to cognitive domains. Whilst some sub-tasks appeared to be associated with specific forms of cognition that does not mean that one sub-task is exclusively linked to only one form of cognition, but that a particular 
area of cognition was more prominent. Indeed, all forms of cognition are interlinked, as Marson (2016) also outlines in an overview of conceptual models of finance management. Memory was one of the primary clusters that transpired, whilst sub-tasks could be divided into different memory types. Longterm (forgetting previously known telephone numbers) and short-term (forgetting what s/he was currently doing) memory could in several activities be linked to the most impaired sub-task. Whilst literature suggests that all three forms of memory are impaired to different degrees in the early stages of dementia (Giebel et al., 2015), evidence linking different types of memory with everyday functioning is very sparse and lacks intricate details. Studies by Schmitter-Edgecombe and colleagues (McAlister and Schmitter-Edgecombe, 2016; Schmitter-Edgecombe and Parsey, 2014; Schmitter-Edgecombe et al., 2009) for example showed how delayed memory, temporal order memory, and prospective memory are related to everyday functioning. However, the majority of research only explores global IADL functioning, although Schmitter-Edgecombe et al. (2009) showed how prospective memory was specifically associated with performing medication management and household activities, and memory for temporal order with medication management and preparing a meal. The deconstruction of activities in the present study adds further knowledge to these individual relationships, as one of the main areas of impairment for cleaning in this study was that PwD forgot it was time to do tasks. This aligns with the prospective memory link evidenced in the study by Schmitter-Edgecombe et al. (2009). Furthermore, when preparing cold meals, PwD primarily struggled with preparing only very simple meals. Although the wrong order of meal preparation was impaired the least, this is likely because PwD were more inclined to only prepare very simple meals, which would have fewer steps than preparing a complex meal. This finding again corroborates the association found between (temporal order) memory and food preparation in the above study. Thus, breaking down activities into its components has obvious benefits for the assessment of cognition and everyday functioning, and its management.

Sub-tasks could also be clustered based on other forms of cognition, including executive functioning and attention. There has been some research into the contributions of the former to performing specific everyday activities. Razani and colleagues (2007) showed how executive functioning contributes to successfully performing finance management, shopping, and transport. In patients with brain damage, deficits with executive functioning have been shown to be able to be rehabilitated, which had a positive effect on meal preparation abilities (Levine et al., 2000). Considering that executive functioning can be rehabilitated in people with Alzheimer's disease also (Cipriani et al., 2006), the present study adds to previous findings recognising the importance of targetedly focusing on this type of cognition.

Similarly, attention appears to be of importance in the performance of several sub-tasks. However, previous research has been very limited in exploring the contribution of attention (Yamin et al., 2016). Out of the five explored forms of cognition in the present study, attention showed fewer links with the traditional IADLs, but more with newly added IADLs on the R-IDDD2, including monitoring own day and monitoring a current activity. The link between driving and attention argued in the present study is supported by previous research in older adults (Munro et al., 2010). Considering that everyday activities are linked to a mix of cognitive areas and that different forms of cognition are 
interlinked with one another, targeting non-memory areas such as attention and executive functioning might result in improvements in a range of activities and possibly even on memory areas. More importantly, having deconstructed IADLs into their sub-tasks has shown how several activities appear to be linked to the same form of cognition. This may imply that providing cognitive training for attention in general may have a positive impact on all activities involving attention, but also other types of cognition which are linked to attention. With little literature on targeted cognitive rehabilitation training in dementia (Bahar-Fuchs et al., 2013), directly targeting specific sub-tasks by relying on their links to cognition is an important step in the intervention arena made possible by deconstructing everyday activities.

Whilst cognition plays a vital role in the successful performance of everyday activities, there are other factors that influence performance. Some sub-tasks might not be relying so much on cognition but to a greater extent on other influences, such as 'requiring help to put on clothes' (Dressing) or 'not being thorough enough' (Washing). For this reason, 11 of the 60 sub-tasks could not be categorised into either of the five cognitive clusters. There may be an element of lacking social awareness, in that the PwD is less aware of what is considered appropriate in personal hygiene. However, particularly in the elderly population, there may be some physical and sensory problems and disabilities that might make it difficult for a PwD to take a shower every day, or to get dressed in the morning. Future research also might consider exploring whether ethnicity contributes to certain sub-tasks, information which was not collected as part of this study. Potentially, different ethnic groups have different perceptions of which activities need to be performed in old age still, as in some ethnic groups younger generations can take over meal preparations or cleaning duties regardless of the level of ability of the older person or PwD.

\section{Conclusions}

This study has important implications for clinical practice. The R-IDDD2 allows the in-depth investigation of everyday functioning deficits in the early stages of dementia. Even if PwD display little to no difficulties with an overall activity, assessing the activity's sub-tasks can help highlighting small deficits. This can contribute to improved recognition of this symptom and thus aid timelier diagnosis in clinical practice. Using the R-IDDD2 can also be useful in care management planning. It is important for carers to only help with those sub-tasks that are impaired, but leave the PwD to continue performing those sub-tasks that are relatively spared. Future research can use the R-IDDD2 to further explore the relationship between cognition and everyday functioning, an area receiving increasingly more attention due to its implication for the development of cognitive interventions.

\section{Description of authors' roles}

C.G. designed the study, was responsible for data collection, analysed the data, and wrote the manuscript. C.G. and D.M. designed the questionnaire. D.M. provided feedback on drafts of the manuscript. 


\section{Conflict of interest}

None.

\section{Funding Sources}

This article presents independent research supported by the National Institute for Health Research (NIHR), under its Programme Grants for Applied Research programme (Grant Reference Number: DTC-RP-PG-0311-12003), and forms part of the doctoral thesis of CG.

\section{Acknowledgements}

We wish to thank staff at the following NHS Trusts for their support in recruiting participants: Catherine Turton, Joanne Woodward at 5 Boroughs Partnership; Ritchard Ledgerd, Alexandra Feast, Charlotte Stoner, Dr Joanne Rodda at North East London; Paula Harman, Dr Farhad Huwez at Southend University Hospital; Joanne Tomkins, Laura Lord, Dr Tarik Qassem at Black Country Partnership; Olga Balazikova, Sarah Hamilton, Ramin Nilforooshan at Surrey and Borders Partnership; Lisa WilkinsonGuy, Prof George Tadros at Heart of England; and Kim Thompson, Leigh Franks, Dr Rashi Negi at South Staffordshire and Shropshire Healthcare. We also wish to thank Division 4 Greater Manchester NIHR Clinical Research Network for recruiting participants to this study through Manchester Mental Health and Social Care and Greater Manchester West NHS Trusts. Our thanks also extend to the JoinDementiaResearch Network for allowing us to recruit carers. 


\section{REFERENCES}

Bahar-Fuchs, A., Clare, L., and Woods, B. (2013). Cognitive training and cognitive rehabilitation for persons with mild to moderate dementia of the Alzheimer's or vascular type: a review. Alzheimer's Research \& Therapy, 5, 35-48.

Bier, N., Bottari, C., Hudon, C., Joubert, S., Paquette, G., and Macoir, J. (2013). The impact of semantic dementia on everyday actions: evidence from an ecological study. Journal of the International Neuropsychological Society, 19(2), 162-172.

Chiong, W., Hsu, M., Wudka, D., Miller, B.L., and Rosen, H.J. (2014). Financial errors in dementia: Testing a neuroeconomic conceptual framework. Neurocase, 20(4), 389-396.

Cipriani, G., Bianchetti, A., and Trabucchi, M. (2006). Outcomes of a computer-based cognitive rehabilitation program on Alzheimer's disease patients compared with those on patients affected by mild cognitive impairment. Archives of Gerontology and Geriatrics, 43(3), 327-335.

Department of Health (2015). The Prime Ministers Challenge on Dementia 2020. London: Department of Health.

Earnst KS, Wadley VG, Aldridge TM, Steenwyk AB, Hammond AE, Harrell LE, and Marson DC (2001). Loss of financial capacity in Alzheimer's disease: The role of working memory. Aging, Neuropsychology, and Cognition, 8(2), 109-119.

Gelinas, I., Gauthier, L., McIntyre, M., et al. (1999). Development of a functional measure for persons with Alzheimer's disease: the disability assessment for dementia. American Journal of Occupational Therapy, 53, 471-481.

Giebel, C.M., Callis, D., and Montaldi, D. (2015). Understanding the cognitive underpinnings of functional impairments in early dementia: a review. Aging \& Mental Health, 19(10), 859-875.

Giebel, C.M., Challis, D., and Montaldi, D. (2016a). A revised Interview for deterioration in daily living activities in dementia reveals the relationship between social activities and well-being. Dementia, 15(5): 1068-1081.

Giebel, C., Challis, D., and Montaldi, D. (2016b). The newly revised Interview for Deteriorations in Daily Living Activities in Dementia (R-IDDD2): Distinguishing initiative from performance at assessment. International Psychogeriatrics, doi: 10.1017/S1041610216002003

Giovannetti, T., Schmidt, K.S., Gallo, J.L., Sestito, N., and Libon, D.J. (2006). Everyday action in dementia: Evidence for differential deficits in Alzheimer's disease versus subcortical vascular dementia. Journal of the Neuropsychological Society, 12(1), 45-63.

Giovannetti, T., Seligman, S.C., Britnell, P., Brennan, L., and Libon, D.J. (2015). Differential effects of goal cues on everyday action errors in Alzheimer's disease versus Parkinson's disease dementia. Neuropsychology, 29(4), 592-602.

Griffith, H.R., Belue, K., Sicola, A., et al. (2003). Impaired financial abilities in mild cognitive impairment: A direct assessment approach. Neurology, 60(3), 449-457.

Levine, B., Robertson, I.H., Clare, L. et al. (2000). Rehabilitation of executive functioning: An experimental-clinical validation of Goal Management Training. Journal of the International Neuropsychological Society, 6(3), 299-312.

Marson DC, et al. (2000). Assessing financial capacity in patients with Alzheimer's disease: A conceptual model and prototype instrument. Archives of Neurology, 57, 877-884.

Marson, D. (2016). Conceptual Models and Guidelines for Clinical Assessment of Financial Capacity. Archives of Clinical Neuropsychology, doi: 10.1093/arclin/acw052.

Martyr, A., \& Clare, L. (2012). Executive function and activities of daily living in Alzheimer's disease: a correlational meta-analysis. Dementia and Geriatric Cognitive Disorders, 33(2-3), 189-203.

McAlister, C., and Schmitter-Edgecombe, M. (2016). Everyday functioning and cognitive correlates in healthy older adults with subjective cognitive concerns. The Clinical Neuropsychologist, DOI:10.1080/13854046.2016.1190404

Mioshi, E., Kipps, C.M., Dawson, K., Mitchell, J., Graham, A., \& Hodges, J.R. (2007). Activities of daily living in frontotemporal dementia and Alzheimer disease. Neurology, 68(24), 2077-2084.

Monaci, L., and Morris, R.G. (2012). Neuropsychological screening performance and the association with activities of daily living and instrumental activities of daily living in dementia: baseline and 18- to 24-month follow-up. International Journal of Geriatric Psychiatry, 27(2), 197-204.

Munro, C.A., Jefferys, J., Gower, E.W., et al. (2010). Predictors of Lane-Change Errors in Older Drivers. Journal of the American Geriatrics Society, 58(3), 457-464.

Nagaratnam, N., Nagaratnam, K., \& O'Mara, D. (2013). Bayer activities of daily living scale in mild and moderate dementia of the Alzheimer type. American Journal of Alzheimer's Disease and Other Dementias, 28, 784-789. 
O'Connor, C.M., Clemson, L., Hornberger, M., et al. (2016). Longitudinal change in everyday function and behavioral symptoms in frontotemporal dementia. Neurology Clinical Practice, doi: 10.1212/CPJ.0000000000000264

Patterson, M.B., Mack, J.L., Neundorfer, M.M., et al. (1992). Assessment of functional ability in Alzheimer disease: a review and a preliminary report on the Cleveland Scale for Activities of Daily Living. Alzheimer Disease and Associated Disorders, 6, 145-63.

Pocnet, C., Rossier, J., Antonietti, J.-P. and von Gunten, A. (2013). Personality features and cognitive level in patients at an early stage of Alzheimer's disease. Personality and Individual Differences, 54(2), 174-179.

Razani, J., Cassas, R., Wong, J.T., et al. (2007). Relationship between Executive Functioning and Activities of Daily Living in Patients With Relatively Mild Dementia. Applied Neuropsychology, 14(3), 208-214.

Schmitter-Edgecombe, M., and Parsey, C.M. (2014). Cognitive Correlates of Functional Abilities in Individuals with Mild Cognitive Impairment: Comparison of Questionnaire, Direct Observation, and Performance-Based Measures. The Clinical Neuropsychologist, 28(5), 726-746.

Schmitter-Edgecombe, M., Woo, E., and Greeley, D.R. (2009). Characterizing Multiple Memory Deficits and Their Relation to Everyday Functioning in Individuals With Mild Cognitive Impairment. Neuropsychology, 23(2), 168-177.

Schwartz, M.F., Buxbaum, L.J., Ferraro, M., Veramonti, T., and Segal, M. (2003). The Naturalistic Action Test. Bury St. Edmunds: Thames Valley Test Company.

Shankle, W.R., Pooley, J.P., Steyvers, M., et al. (2013). Relating Memory To Functional Performance In Normal Aging to Dementia Using Hierarchical Bayesian Cognitive Processing Models. Alzheimer Disease and Associated Disorders, 27(1), 16-22.

Sikkes, S.A.M., Visser, P.J., Knol, D.L., et al. (2011). Do instrumental activities of daily living predict dementia at 1- and 2-year follow-up? Findings from the development of screening guidelines and diagnostic criteria for predementia Alzheimer's disease study. Journal of the American Geriatrics Society, 59(12), 2273-2281.

Teunisse, S., Derix, M.M.A. and van Crevel, H. (1991). Assessing the severity of dementia. Archives of Neurology, 48(3), 274-277.

van Steenoven I, Aarsland D, Hurtig H, et al. (2014). Conversion between mini-mental state examination, Montreal Cognitive Assessment, and Dementia Rating Scale-2 scores in Parkinson's disease. Mov. Disord, 29(14), 1809-1815.

Vermeersch, S., Gorus, E., Cornelis, S. and De Vriendt, P. (2015). An explorative study of the relationship between functional and cognitive decline in older persons with mild cognitive impairment and Alzheimer's disease. British Journal of Occupational Therapy, 78(3), 166-174.

Yamin, S., Stinchcombe, A., and Gagnon, S. (2016). Deficits in Attention and Visual Processing but not Global Cognition Predict Simulated Driving Errors in Drivers Diagnosed with Mild Alzheimer's Disease. American Journal of Alzheimer's Disease \& Other Dementias, 31(4), 351-360.

Zanetti, O., Geroldi, C., Frisoni, G.B., Bianchetti, A., \& Trabucchi, M. (1999). Contrasting Results Between Caregiver's Report and Direct Assessment of Activities of Daily Living in Patients Affected by Mild and Very Mild Dementia: The Contribution of the Caregiver's Personal Characteristics. Journal of the American Geriatrics Society, 47(2), 196-202. 